This forum is dedicated to personal health in all its many facets: decision making, goal setting, celebration, discovery, reflection, and coordination, among others. We look at innovations in interactive technologies and how they help address current critical healthcare challenges. - Gillian R. Hayes, Editor

\title{
Expanding Design Possibilities for Life with Dementia
}

Amy S. Hwang, University of Toronto and Toronto Rehabilitation Institute

Khai N. Truong, University of Toronto

Alex Mihailidis, University of Toronto and Toronto Rehabilitation Institute

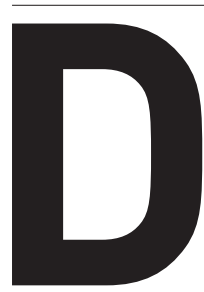

ementia - a syndrome most commonly caused by Alzheimer's disease-is widely understood as progressive memory loss and functional decline in older adults. Still without a cure, dementia conjures images of loss, deterioration, and dependency on caregivers - usually family members - until care needs warrant institutionalization. Persons with dementia need increasing care, and caregivers need support to cope with the increasing burden.

This biomedical construction of dementia - considered an instance of the broader biomedicalization of aging, eloquently critiqued by Vines et al. [1]has spawned significant investments in "gerontechnological innovations" [2]. These strive to promote independence and dignity for persons with dementia by delivering cognitive training (e.g., serious games), assisting cognition in everyday activities (e.g., delivering context-aware prompts and reminders), and ensuring safety and well-being (e.g., activity monitoring). Meanwhile, they aim to afford peace of mind, provide practical care assistance, and reduce the workload of caregivers. Our own previous research that explored and co-designed ambient assistive living interfaces with family care partners exemplified these shared goals [3].

Such innovations, however, remain largely unrealized despite the touted “triple win” [2] to older adults, industry, and society. Explanations for this disappointment include limited funding to commercialize research, lack of academic and industry collaborations, and barriers to market entry for health interventions. Economies of scale have also remained unrealized owing to low consumer awareness, high costs, and technology abandonment, often attributed to poor design. Based on our empirical work and inspiration from other scholars (in particular, [1] and [4]), we posit a different perspective on the problem: There is a fundamental incongruence in the design of rational, mechanistic health solutions for irrational, complex social problems engendered by life with dementia.

In this article, we share insights from the past five years of qualitative and design research that have disrupted our own biomedical understanding of the "dementia problem" and, in turn, profoundly shifted our design perspective. Our insights are grounded in our empirical work and reflect current discussions within dementia and gerontology scholarship. Fueled by growing activism among persons and families with dementia, these ideas are now permeating public awareness through mass campaigns, such as

\footnotetext{
Insights

$\rightarrow$ Rational, mechanistic health solutions are incongruent with the irrational, complex social problems engendered by life with dementia.

$\rightarrow$ Empowering persons and families with dementia means understanding their experiences beyond their symptoms, problems, and burdens.

$\rightarrow$ Seeing care as a way of relating can expand our scope of design possibilities.
}

Still Here by the Alzheimer's Society of Canada, that combat the stigma associated with dementia. Riding this wave of social change, we hope to inspire new design thinking based on a richer appreciation of the social relational realities that have long been marginalized to issues of cause, cure, and dementia-care management.

Insight \#1. Living with early-stage dementia can bring about social tensions that in turn result in people resisting care and support, despite the apparent logic. Throughout our work, family members shared stories about their help and concern being resisted, refused, or unacknowledged by relatives/spouses with dementia. In workshops with persons with mild dementia, we observed in several participants tendencies to steer conversations away from discussing "deficiencies" to joking playfully about their forgetfulness, exchanging tips with peers as a means of avoiding asking family members for help, and emphasizing examples of mastery and continued abilities. Continuing habituated activities (also found by [5]), solving one's own problems, and mastering new skills meant maintaining one's sense of self. These ways of continuing to be somebody - someone recognized, valued, and needed - in one's family and community can be threatened when a person feels overprotected or infantilized by family members.

Seemingly in resistance, people persist as best they can with their activities, commitments, and, interestingly, their technology use. For instance, a reminder alarm for a 


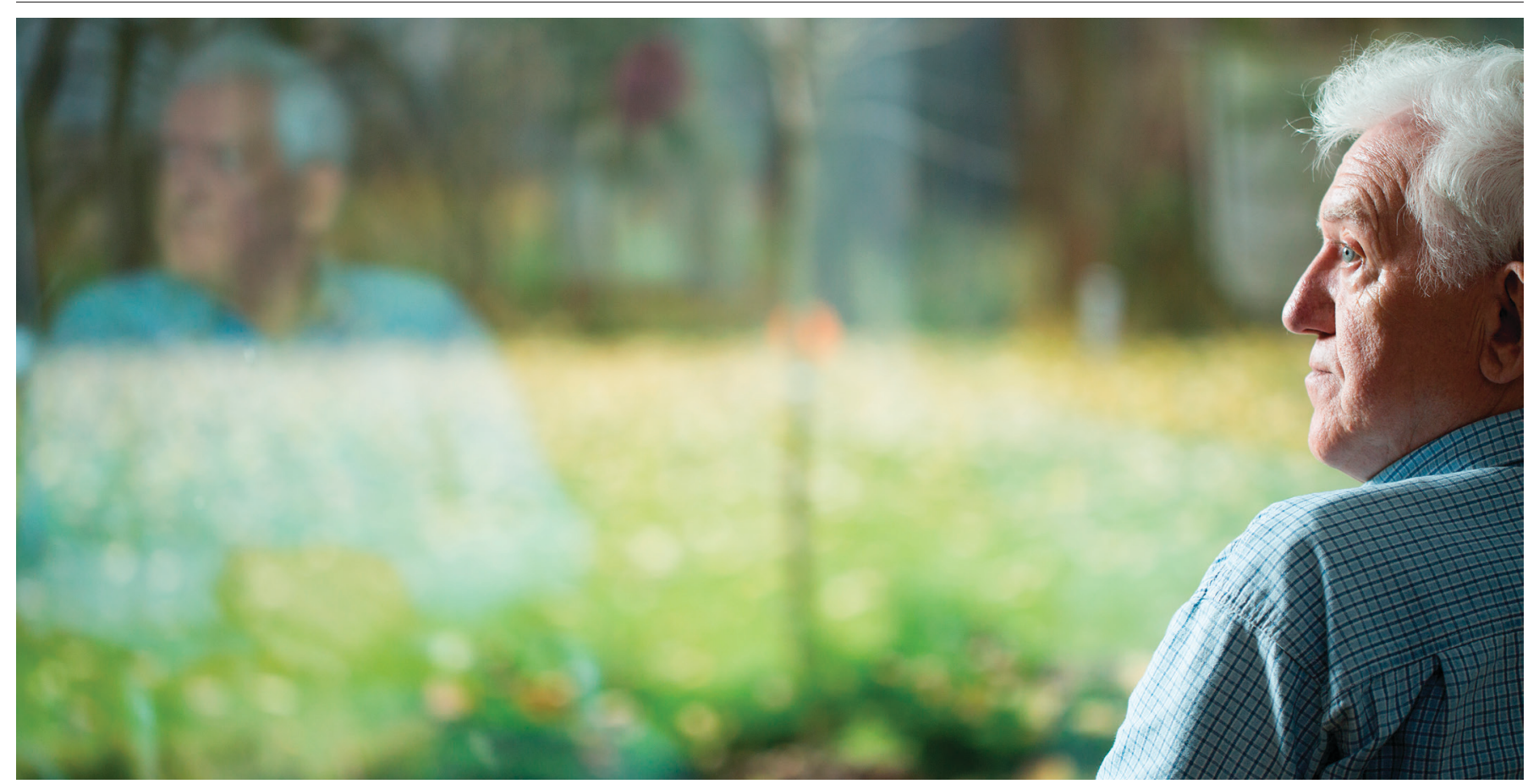

menial task set by a worrying family member was perceived in one of our studies as overprotective. Yet, when initiated by the person with dementia, the "solution" not only solved a rational problem, but it also demonstrated to his family his continuing competence, creativity, and mastery.

Although such issues of self-identity and social stigma have been examined in gerontology and dementia scholarship, they remain unaddressed by the myriad emerging technologies that prize care efficiency over human empathy. There seems to be a tendency to develop “interventions" that objectify persons with dementia by circumventing their input and overriding their self-initiated strategies. By designing through a lens that sees the disease and overlooks the person living with it, we forget that products say something about the user. When the features and functionalities we design strip persons with dementia of their meaningful activities, we risk disempowering the very people we intended to empower.

Insight \#2. Further complicating matters is the complexity of family care experiences, which the biomedical discourse tends to reduce to a singular label: burden. In our ambient assistive living study, we expected family care partners to embrace a system to which they could delegate selected care activities that they deemed menial or burdensome. Instead, we learned that delegating care to another is not always a rational, straightforward decision; care was found to depend on available time, moods, and the personal and relational meanings ascribed to activities [3]. Subsequent focus groups with adult children elaborated our understanding of care experiences [6]. Indeed, burdensome tasks were broached but, more fundamentally, participants discussed role and value conflicts (e.g., taking care of one's parents and children as part of the "sandwich generation"); tension and sometimes irreconcilable conflict with siblings over care decisions; fear and mistrust in residential care institutions; invisible barriers to social service access; and unrelenting feelings of guilt despite tremendous, mounting personal sacrifice. Simultaneously, however, care experiences embodied and reinforced collective values, personal growth, and a sense of renewed purpose across participants. The idea of burden captures but one slice of the complex care experience and, thus, designing

\section{The idea of burden captures but one slice of the complex care experience.}

to alleviate burden stops short of a multitude of other design opportunities that become possible when we can appreciate this complexity.

Insight \#3. Care relationships and practices cannot be reduced and mechanized to acts of giving and receiving; caring should be understood as a way of relating that ebbs, flows, and transforms in the context of dementia. Persons with dementia and their family members may align on certain care practices, while others may provoke social friction. These call for negotiation, where social boundaries are in constant motion as people adapt to changes in ability, social roles, and relationships. Persons with dementia may draw invisible boundaries when they choose to share or conceal their everyday problems, or selectively seek help. They may then adjust these boundaries in response to family members' reactions, judgments, or behaviors. For instance, an isolated memory lapse that initiates protective behaviors from a spouse may create a reluctance to share one's frustrations openly. In turn, social withdrawal may lead the spouse to worry even more. With time, the spouse may adjust her approach and create space for the person with dementia to express vulnerability once again. As these ways of relating ebb and flow, we must conceptualize care as a motion picture 
of human experience that cannot be reduced to a set of still images capturing problems and burdens.

Designing for relating. Embracing Peter Wright and John McCarthy's experience-centered design [4] paradigm, we concur that design has much to gain from interpretive and critical qualitative approaches that can produce more generative understandings of experience in its multiplicity, complexity, and particularity. At the heart of experience-centered design is an appreciation for the richness of human experience, a concern for meaning and how people make sense of their experiences, and the mindset that innovation should strive to enhance life, particularly for those who are disenfranchised.

From our perspective, doing experience-centered design means examining what might be taken for granted when we study our intended consumers. Design research should strive to not only describe human needs and behaviors at face value, but also to understand the fundamental values, processes, and mechanisms that underlie them. In doing so, we might improve support for current behavior while making better estimations about future behavior-how people will act differently around and through the use of our designed innovations. Socializing these ways forward with industry designers and innovators, we advocate for the value of synergistic collaboration with anthropologists, social gerontologists, and clinical scientists who are aligned with more social models of disability.

We also generally encourage all designers, innovators, and professionals to critically consider what their design concepts, products, and services say about the people who are expected to benefit from them. What messages do our innovations convey about the users? Do they confer meanings that fit with how people want others to relate to them? From whose perspective is the "solution" being designed and appropriated vis-à-vis who is expected to use the innovation? We should acknowledge that the market plays a powerful role in creating and reinforcing social images through advertising. We call upon industry professionals to be mindful of the detrimental impact that inadvertent stereotyping can have on the intended consumers.

Lastly, instead of touting "solutions" that need validation - as the biomedical view would encourage - perhaps we might also consider a humbler position and think about our designs as possibilities in need of collaboration and appropriation with our intended stakeholders. Collaboration should aim for empathy and dialogue, central tenets of experience-centered design [4], and employ methods that co-construct how people relate to one another and to the proposed innovations. We have found that naturalistic field studies make visible the social relations that influence and are impacted by the innovation in question. We encourage field studies that investigate people's intuitive and improvisational strategies for managing everyday life with dementia. Here, we emphasize that these strategies often entail social relationships and processes, which prompt us to examine these ways of relating as the unit of analysis, instead of more traditional approaches that study a user's needs and, separately, his context. In our current study, for instance, we are exploring how couples and families living with dementia can be supported by a community program to adopt off-the-shelf technology. In attending closely to how people relate to one another and their new technologies in this context, we have been amazed to learn how their relationships, support practices, and even program development has been transformed through these social experiences.

As designers and innovators, we can challenge the disease-intervention/ problem-solution paradigm and join the social dementia movement that is among us. Through our design ideas, processes, and products, we can protect meaningful human experiences from being buried in the rationality of clinically or technologically validated "solutions." By foregrounding ways in which people relate to themselves, each other, and the world, we not only begin to watch the motion picture of living and relating with dementia, but we also we carve a place for our innovations within it.

\section{ENDNOTES}

1. Vines, J., Pritchard, G., Wright, P., Olivier, P., and Brittain, K. An age-old problem. ACM Trans. on Computer-Human Interaction 22, 1 (Mar. 2015), 1-27.

2. Neven, L. By any means? Questioning the link between gerontechnological innovation and older people's wish to live at home. Technological Forecasting (t) Social Change 93 (Apr. 2015), 32-43.

3. Hwang, A.S., Truong, K.N., Cameron, J.I., Lindqvist, E., Nygård, L., and Mihailidis, A. Co-designing ambient assisted living (AAL) environments: Unravelling the situated context of informal dementia care. BioMed Research International, 2015.

4. Wright, P.C. and McCarthy, J. ExperienceCentered Design: Designers, Users, and Communities in Dialogue. Synthesis Lectures on Human-Centered Informatics 3, 1 (2010), 107-123.

5. Phinney, A., Chaudhury, H., and O'Connor, D.L. Doing as much as I can do: The meaning of activity for people with dementia. Aging (t) Mental Health 11, 4 (2007), 384-393.

6. Hwang, A.S., Rosenberg, L., Kontos, P.C., Mihailidis, A., and Nygård, L. Beyond the discourse of 'burden': Exploring the experiences of adult children who care for parents with dementia. Presented at: Life with Dementia: Relations. Linköping University, Norrköping, Sweden, 2014.

(2) Amy S. Hwang is a doctoral candidate at the Rehabilitation Sciences Institute at the University of Toronto, Toronto Rehab, and a trainee of Canada's AGE-WELL Network of Centres of Excellence. Her work explores how technological innovation can be designed to enhance experiences for persons and families living with dementia.

$\rightarrow$ amy.hwangamail.utoronto.ca

(1) Khai N. Truong is an associate professor in the Department of Computer Science at the University of Toronto. His research investigates tools and methods to support the development of novel ubiquitous computing systems and techniques to facilitate user interactions with off-the-desktop computing devices and services.

$\rightarrow$ khaidacs.toronto.edu

(1) Alex Mihailidis is the Barbara G. Stymiest Research Chair in Rehabilitation Technology at University of Toronto and Toronto Rehab, and Scientific Director of Canada's AGE-WELL Network of Centres of Excellence. His research over the past 15 years has investigated intelligent home and health systems with a focus on elder care.

$\rightarrow$ alex.mihailidisQutoronto.ca

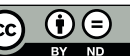

This work is licensed under a Creative Commons Attribution-NoDerivs International 4.0 License. 